\title{
Dynamic double layer force between charged surfaces
}

\author{
Bhavya Balu $0^{*}$ and Aditya S. Khair (1) \\ Department of Chemical Engineering, Carnegie Mellon University, Pittsburgh, Pennsylvania 15213, USA
}

(Received 12 December 2019; accepted 9 January 2020; published 7 February 2020)

\begin{abstract}
We develop a theory for the "dynamic double layer force" between charged surfaces in an electrolyte under a time-dependent voltage. Specifically, the force between two planar surfaces is calculated within the PoissonNernst-Planck framework for dilute electrolytes, accounting for unequal ionic diffusivities. Due to the inherent nonlinear dependence of the force on the electric potential, a sinusoidal voltage oscillating with frequency $\omega$ gives rise to a nonzero time-averaged force, along with a component oscillating with frequency $2 \omega$. The timeaveraged force is always attractive, as expected for surfaces of opposite polarity. However, the instantaneous force switches between attractive and repulsive over an oscillation cycle at certain frequencies. Next, the force is quantified for suddenly applied and pulsed voltages. In the former case, the force approaches its long-time limit exponentially: on a resistor-capacitor or bulk diffusion time scale for an electrolyte with equal or unequal ionic diffusivities, respectively. The long-time decay of the force for a pulsed voltage also decays exponentially on the same timescales. The theory developed here is a dynamic generalization of the equilibrium double layer force, with potential applications to electrochemical devices, force-based spectroscopy, and colloidal directed assembly.
\end{abstract}

DOI: 10.1103/PhysRevResearch.2.013138

\section{INTRODUCTION}

Charged surfaces in contact with electrolytes are abundant in electrochemical [1], colloidal [2], and biological [3] systems. The ions in the electrolyte form a diffuse charge layer that screens the surface charge. The thickness of this layer for sufficiently dilute solutions is characterized by the Debye length, $\kappa^{-1}=\sqrt{\varepsilon k_{B} T /\left(2 I_{0} e^{2}\right)}$, where $\varepsilon$ is the permittivity of the electrolyte, $k_{B}$ is the Boltzmann constant, $T$ is the temperature, $I_{0}$ is the ionic strength, and $e$ is the charge on a proton. The net (counterion) charge density results in an electrical body force on a fluid element within the layer, which must be balanced by an osmotic pressure gradient to maintain mechanical equilibrium. The presence of such diffuse layers implies that the interaction between charged surfaces in an electrolyte is fundamentally different to that in a dielectric medium. Quantifying this interaction is crucial in predicting the macroscopic stability of dispersions [4,5], designing electrochemically active devices [6-8], and engineering self- and directed assembly of colloids and macromolecules $[9,10]$.

The seminal Derjaguin-Landau-Verwey-Overbeek (DLVO) theory [2] describes the long range electrostatic and short range van der Waals forces between charged surfaces at thermodynamic equilibrium. State of the art experimental techniques $[4,11]$ use it to analyze measurements of the force between charged surfaces. The DLVO theory needs

\footnotetext{
*bbalu@andrew.cmu.edu

Published by the American Physical Society under the terms of the Creative Commons Attribution 4.0 International license. Further distribution of this work must maintain attribution to the author(s) and the published article's title, journal citation, and DOI.
}

modification to account for dynamic interactions that may arise due to charge regulation [12-14], electrokinetic particle motion [15-17], and external charging of electrochemical cells [7,18-20]. Research on charge regulation models focuses on systems in quasiequilibrium; i.e., the ions in the electrolyte reach their equilibrium distribution much faster than a charge regulating reaction on the surface. Studies on the effect of electrokinetic motion focus on the modified hydrodynamic force due to the relative motion of the ions in the diffuse layer and the surface.

Recent surface force apparatus experiments by PerezMartinez and Perkin [7] externally apply an oscillating voltage and simultaneously measure the time-dependent surface force between two electrodes across an electrolyte solution. Tivony et al. [20] measure the time-dependent surface force due to a suddenly applied, or step, voltage in a similar apparatus. Motivated by these recent studies, here we develop a theoretical model for the "dynamic double layer force" between two surfaces whose potential difference oscillates in time. We also consider the response of the system to a suddenly applied voltage and a single voltage pulse. Our focus is on quantifying how the ion transport across the electrolyte, via electromigration and diffusion, determines the time-dependent force on the surface. A recent study [21] calculated the double layer force between two planar surfaces in a symmetric, binary electrolyte under an ac voltage with a dc bias. Here, we consider an asymmetric electrolyte with unequal ionic diffusivities and calculate the force for a suddenly applied dc voltage and a voltage pulse in addition to an ac voltage. We do not consider the effect of surface reactions; thus the time dependence solely arises from the dynamic formation of double layers in response to the external voltage. Our theory contributes a dynamic, outof-thermodynamic-equilibrium generalization of the classical 


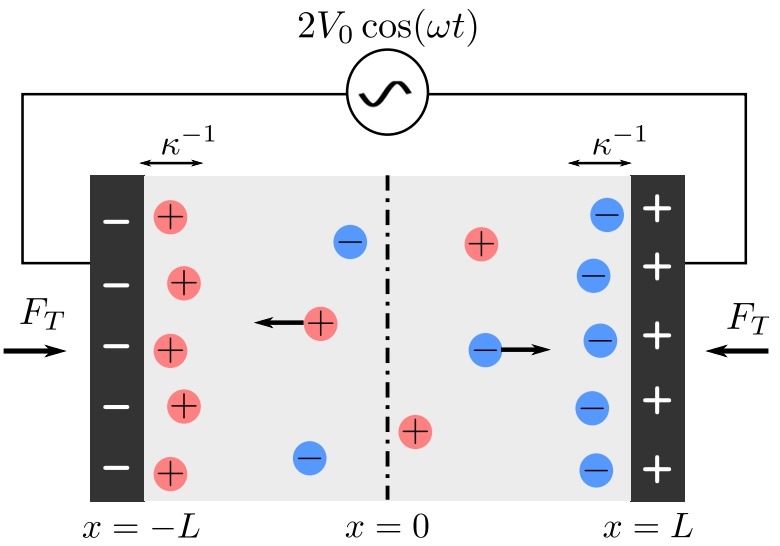

FIG. 1. Schematic of a model electrochemical cell under an oscillating voltage $2 V_{0} \cos (\omega t)$. A time dependent force of magnitude $F_{T}$ is exerted on each electrode due to the ion transport under this potential difference. Regions of net ionic charge are confined to a distance of the order of the Debye length, $\kappa^{-1}$, from each electrode. Typically, $\kappa^{-1}$ is much smaller than the distance between electrodes, $L$.

electrostatic double layer force on which DLVO theory is founded.

We show that the force per unit area on a surface scales as $\varepsilon V^{2} / L^{2}$, where $\varepsilon$ is the permittivity of the electrolyte, $V$ is the applied voltage, and $L$ is the distance between the electrodes. Thus taking $V=k_{B} T / e \approx 25 \mathrm{mV}$ at $298 \mathrm{~K}$ across $L=1 \mu \mathrm{m}$ in an aqueous electrolyte generates a stress of about $1 \mathrm{~Pa}$. This stress exerts a force of $O(10 \mathrm{pN})$ on a spherical particle of one micron radius, which is a typical force scale in colloidal and biological soft matter. Further, the stress is comparable to the elastic modulus of a nervous membrane [22]. We suggest, therefore, that the dynamic double layer force is relevant in soft materials, for example, in biocompatible flexible electronics that aim to mimic the mechanical properties of biological membranes [23]. Further, our analysis could be used to develop new experimental techniques, such as a force based spectroscopy, in analogy to electrochemical impedance spectroscopy, to probe physicochemical electrolyte properties. Finally, we show that the dynamic double layer force can vary by orders of magnitude with frequency. Hence estimation of the mechanical force on electrochemically active surfaces via DLVO theory can lead to wildly erroneous results.

The system considered here represents a minimal model for dynamic forces between charged surfaces exposed to time varying voltages. Our work serves as a starting point to investigate more complex systems that have temporally varying surface charges, where nonideal ion interactions and irregular particle shapes could play a significant role in determining the double layer force.

\section{PROBLEM FORMULATION}

\section{A. Ion dynamics}

We consider a model electrochemical cell with a dilute, monovalent, binary electrolyte between planar, parallel, blocking electrodes (Fig. 1). It is assumed that the electrode dimensions are large compared to the distance $(2 L)$ between them; hence the ion transport is solely in the direction $(x)$ between the electrodes. We study the response of this system to an applied alternating voltage $2 V(t)=2 V_{0} \cos (\omega t)$, where $V_{0}$ is the amplitude, $\omega$ is the angular frequency of the signal, and $t$ represents time. From the solution to this problem, we also derive the response of the cell to a suddenly applied voltage and a pulsed voltage. This effectively allows us to determine the dynamic double layer force between such surfaces.

We assume that $V_{0}$ is small compared to the thermal voltage scale $k_{B} T / e$, such that the dimensionless voltage $\tilde{V}_{0}=V_{0} e / k_{B} T \ll 1$. Henceforth, the tilde decoration denotes a dimensionless variable. We define the charge density $\rho$ and ionic strength $I$ in terms of the ionic concentrations $n_{ \pm}$as $\rho=e\left(n_{+}-n_{-}\right)$, and $2 I=n_{+}+n_{-}$. The normalized charge density $\tilde{\rho}$, ionic strength $\tilde{I}$, and electric potential $\tilde{\phi}$ in the electrolyte are governed by the Poisson-Nernst-Planck (PNP) equations. For our model system, the dimensionless, linearized PNP equations are

$$
\begin{aligned}
\kappa L\left(\frac{\partial \tilde{\rho}}{\partial \tilde{t}}+\frac{D_{A}}{D_{F}} \frac{\partial \tilde{I}}{\partial \tilde{t}}\right) & =\frac{\partial^{2} \tilde{\rho}}{\partial \tilde{x}^{2}}-\kappa^{2} L^{2} \tilde{\rho}, \\
\kappa L\left(\frac{D_{A}}{D_{F}} \frac{\partial \tilde{\rho}}{\partial \tilde{t}}+\frac{\partial \tilde{I}}{\partial \tilde{t}}\right) & =\frac{\partial^{2} \tilde{I}}{\partial \tilde{x}^{2}}, \\
\frac{\partial^{2} \tilde{\phi}}{\partial \tilde{x}^{2}} & =-\kappa^{2} L^{2} \tilde{\rho} .
\end{aligned}
$$

The first two equations arise from linear combinations of the cationic and anionic species balances [24,25], and the third is Poisson's equation describing the variation in the local electric field due to the ionic space charge density. A derivation of (1) is presented in Appendix A. Here, $D_{A}=2 D_{+} D_{-} /\left(D_{+}+D_{-}\right)$ is the ambipolar diffusivity, $D_{F}=2 D_{+} D_{-} /\left(D_{-}-D_{+}\right)$, and $D_{+}$and $D_{-}$are the (distinct) diffusivities of the cations and anions, respectively. Note, we do not assume ambipolar diffusion. The ambipolar diffusion coefficient $D_{A}$ naturally arises when expressing the PNP equations (1) in terms of the charge density and ionic strength, rather than the individual ion concentrations $n_{ \pm}$. The charge density is normalized by $2 I_{0} e$, and the ionic strength by $I_{0}$, where $I_{0}$ is the equilibrium ionic strength when there is no applied voltage, the potential by $k_{B} T / e$, the distance by $L$, the length of the half cell, and the time by the resistor-capacitor (RC) charging time $\tau_{\mathrm{RC}}=\kappa^{-1} L / D_{A}[18,26]$. The system is subject to boundary conditions at the blocking electrodes, $\tilde{x}= \pm 1$; namely,

$$
\left(\frac{\partial \tilde{\rho}}{\partial \tilde{x}}+\frac{\partial \tilde{\phi}}{\partial \tilde{x}}\right)_{ \pm 1}=0, \quad\left(\frac{\partial \tilde{I}}{\partial \tilde{x}}\right)_{ \pm 1}=0, \quad \tilde{\phi}_{ \pm 1}= \pm \tilde{V}(\tilde{t}),
$$

corresponding to zero flux of charge and neutral salt, and an applied voltage of $\pm \tilde{V}(\tilde{t})$ at opposite electrodes, and hence a net potential difference of $2 \tilde{V}(\tilde{t})$. We consider three forms of $\tilde{V}(\tilde{t})$ : an oscillating voltage, $\tilde{V}=\tilde{V}_{0} \cos (\tilde{\omega} \tilde{t})$, a suddenly applied voltage, $\tilde{V}=\tilde{V}_{0} \mathcal{H}(\tilde{t})$ where $\mathcal{H}(\tilde{t})$ is the Heaviside step function, and a voltage pulse, $\tilde{V}=\tilde{V}_{0} \delta(\tilde{t})$, where $\delta(\tilde{t})$ is the Dirac delta function.

\section{B. Double layer force}

The force on an electrode is calculated as the integral of the traction on its surface originating from osmotic pressure and electrical (Maxwell) stress. For the one-dimensional system 
considered here, the force per unit area, normalized as shown below, is given by

$$
F_{T}\left(\frac{\kappa^{2} L^{2}}{I_{0} k_{B} T}\right) \equiv \tilde{F}_{T}=\left[\tilde{p}(\tilde{x})-\tilde{p}_{0}\right]-\frac{1}{2}\left(\frac{\partial \tilde{\phi}}{\partial \tilde{x}}\right)^{2} .
$$

In the right side of (3), the first term represents the difference in the local osmotic pressure $\tilde{p}$ due to an external voltage and a bulk reservoir value $\tilde{p}_{0}$ under no voltage. This pressure is due to an increase in ionic strength between the electrodes relative to the value $I_{0}$ that defines the (dimensional) reservoir pressure $p_{0}=I_{0} k_{B} T$. The second term in the right side of (3) is due to electric stresses. Note that although the pressure $\tilde{p}$ and the potential $\tilde{\phi}$ vary with location $\tilde{x}$, their combination in (3) is a spatial constant. The scale of the force can be understood as a product of the reservoir osmotic pressure $I_{0} k_{B} T$ and the square of the normalized Debye length $1 /\left(\kappa^{2} L^{2}\right)$. Since the Debye length is inversely proportional to the square root of the ionic strength $I_{0}$, the force scale is independent of $I_{0}$. The permittivity of the electrolyte $\varepsilon$ enters the force scale through the Debye length. We assume that the permittivity of the electrolyte is the same as that of the solvent, neglecting the influence of the ions, which can alter the permittivity [27-29]. It would be an interesting future direction to consider the influence of a frequency or ion-concentration dependent permittivity on the dynamic double layer force. To calculate $\tilde{p}$ we assert that the system is in mechanical equilibrium; thus the pressure gradient is balanced by the divergence of the electric stress. As shown in Appendix B, this leads to an osmotic pressure gradient, $\frac{\partial \tilde{p}}{\partial \tilde{x}}=\frac{1}{2} \frac{\partial}{\partial \tilde{x}}\left(\frac{\partial \tilde{\phi}}{\partial \tilde{x}}\right)^{2}$. Importantly, we do not assume that the ions follow a Boltzmann distribution (as is standard in DLVO theory) since the force we calculate is not at thermodynamic equilibrium.

\section{RESULTS AND DISCUSSION}

\section{A. Force spectroscopy}

For an applied oscillating voltage, $2 \tilde{V}(\tilde{t})=2 \operatorname{Re}\left(\tilde{V}_{0} e^{i \omega \tilde{t}}\right)$, with frequency $\tilde{\omega}$ normalized by $1 / \tau_{\mathrm{RC}}$, we expect the solutions of the linearized PNP equations to be of the form $\tilde{g}=$ $2 \operatorname{Re}\left(\tilde{g}_{1} e^{i \tilde{\omega} \tilde{t}}\right)$, where $\tilde{g}_{1}$ is a complex valued function of $\tilde{x}$ and $\tilde{\omega}, \iota=\sqrt{-1}$, Re is for the real part, and $\tilde{g}$ represents $\tilde{\rho}, \tilde{I}$, or $\tilde{\phi}$. This is equivalent to performing a Fourier transform of the PNP equations and yields the long-time oscillatory response of the electrolyte, i.e., after the initial transients have died out. Details of the solution process are provided in Appendix A. The force on the electrode depends on the electric potential gradient,

$$
\frac{\partial \tilde{\phi}}{\partial \tilde{x}}=2 \operatorname{Re}\left(\frac{\partial \tilde{\phi}_{1}}{\partial \tilde{x}} e^{\imath \tilde{\omega} \tilde{t}}\right),
$$

where the potential gradient in Fourier space is

$$
\begin{gathered}
\frac{\partial \tilde{\phi}_{1}}{\partial \tilde{x}}=\frac{\tilde{V}_{0}}{Q}\left[-\kappa^{2} L^{2}\left(\frac{\cosh \left(\lambda_{1} \tilde{x}\right)}{\lambda_{1} \cosh \left(\lambda_{1}\right)}-\frac{\lambda_{1}}{\lambda_{2}} \frac{v_{2}}{v_{1}} \frac{\cosh \left(\lambda_{2} \tilde{x}\right)}{\lambda_{2} \cosh \left(\lambda_{2}\right)}\right)\right. \\
\left.+\left(\lambda_{1}-\frac{\kappa^{2} L^{2}}{\lambda_{1}}\right)-\frac{\lambda_{1}}{\lambda_{2}} \frac{v_{2}}{v_{1}}\left(\lambda_{2}-\frac{\kappa^{2} L^{2}}{\lambda_{2}}\right)\right] .
\end{gathered}
$$

Here,

$$
\begin{aligned}
Q= & {\left[\left(\lambda_{1}-\frac{\kappa^{2} L^{2}}{\lambda_{1}}\right)-\frac{\lambda_{1}}{\lambda_{2}} \frac{v_{2}}{v_{1}}\left(\lambda_{2}-\frac{\kappa^{2} L^{2}}{\lambda_{2}}\right)\right.} \\
& \left.+\frac{\kappa^{2} L^{2}}{\lambda_{1}^{2}} \tanh \lambda_{1}-\frac{\lambda_{1}}{\lambda_{2}} \frac{v_{2}}{v_{1}} \frac{\kappa^{2} L^{2}}{\lambda_{2}^{2}} \tanh \lambda_{2}\right],
\end{aligned}
$$

and $\lambda_{1}$ and $\lambda_{2}$ are the eigenvalues of the system of ODEs governing charge density and ionic strength $(1) ;\left[v_{1}, 1\right]$ and $\left[v_{2}, 1\right]$ are the corresponding eigenvectors. These are

$$
\lambda_{i}^{2}=\frac{\kappa^{2} L^{2}}{2}+\iota \tilde{\omega} \kappa L \pm \frac{\kappa^{2} L^{2}}{2} \sqrt{\left(1-\frac{4 \tilde{\omega}^{2}}{\kappa^{2} L^{2}} \frac{D_{A}^{2}}{D_{F}^{2}}\right)}
$$

and

$$
\frac{v_{2}}{v_{1}}=\frac{1-\sqrt{\left(1-\frac{4 \tilde{\omega}^{2}}{\kappa^{2} L^{2}} \frac{D_{A}^{2}}{D_{F}^{2}}\right)}}{1+\sqrt{\left(1-\frac{4 \tilde{\omega}^{2}}{\kappa^{2} L^{2}} \frac{D_{A}^{2}}{D_{F}^{2}}\right)}} .
$$

Our Eqs. (5)-(8) are in agreement with Barbero and Lelidis [30] who derive equivalent expressions in their study on ambipolar diffusion in impedance spectroscopy. To proceed, we first obtain the pressure distribution $\tilde{p}(\tilde{x})$ by invoking mechanical equilibrium. Then, the force per unit area $\tilde{F}_{T}$ on the electrode at $\tilde{x}=1$ is calculated using (3). As detailed in Appendix B, this yields a force with a steady, timeindependent component and a component that oscillates with twice the applied frequency, which we partition as

$$
\tilde{F}_{T}=\tilde{F}_{S}+\operatorname{Re}\left(\tilde{F}_{O} e^{2 i \tilde{\omega} \tilde{t}}\right) .
$$

Here, the steady, time-averaged force is

$$
\tilde{F}_{S}=-\left|\frac{\tilde{V}_{0}}{Q}\left[\left(\lambda_{1}-\frac{\kappa^{2} L^{2}}{\lambda_{1}}\right)-\frac{\lambda_{1}}{\lambda_{2}} \frac{v_{2}}{v_{1}}\left(\lambda_{2}-\frac{\kappa^{2} L^{2}}{\lambda_{2}}\right)\right]\right|^{2},
$$

where $|z|$ represents the modulus of the complex number $z$. The oscillating component of the force has amplitude

$$
\begin{aligned}
\tilde{F}_{O}= & -\frac{\tilde{V}_{0}^{2}}{Q^{2}}\left\{\kappa^{4} L^{4} \frac{\operatorname{sech}^{2} \lambda_{1}}{2 \lambda_{1}^{2}}+\frac{\lambda_{1}}{\lambda_{2}} \frac{v_{2}}{v_{1}} \kappa^{4} L^{4} \frac{\operatorname{sech}^{2} \lambda_{2}}{2 \lambda_{2}^{2}}\right. \\
& \left.+\left[\left(\lambda_{1}-\frac{\kappa^{2} L^{2}}{\lambda_{1}}\right)-\frac{\lambda_{1}}{\lambda_{2}} \frac{v_{2}}{v_{1}}\left(\lambda_{2}-\frac{\kappa^{2} L^{2}}{\lambda_{2}}\right)\right]^{2}\right\} .
\end{aligned}
$$

Thus there is a nonzero time averaged force over an oscillation cycle even though the applied voltage averages out to zero. This is due to the inherently nonlinear (quadratic) dependence of force on the applied potential. Note that because we consider a purely oscillating field with no dc bias, our expression for force has no component that oscillates with a frequency $\tilde{\omega}$, which is different from a recent calculation [21], where a dc bias is considered. The total force $\tilde{F}_{T}$ as a function of time is plotted in Fig. 2(a) for small, intermediate, and large frequencies $\tilde{\omega}$. The dependence of the maximum value of the force during an oscillation cycle on the normalized inverse Debye length $\kappa L$ and on the applied frequency $\tilde{\omega}$ is plotted in Figs. 2(b) and 2(c). The dependence of the time averaged force on $\kappa L$ and $\tilde{\omega}$ is shown in Fig. 3.

At small frequencies, $\tilde{\omega} \rightarrow 0$, with $\kappa L$ fixed, the amplitude of oscillations $\left|\tilde{F}_{O}\right| \rightarrow \tilde{V}_{0}^{2} \kappa^{2} L^{2} \operatorname{cosech}^{2}(\kappa L)$, which precisely 

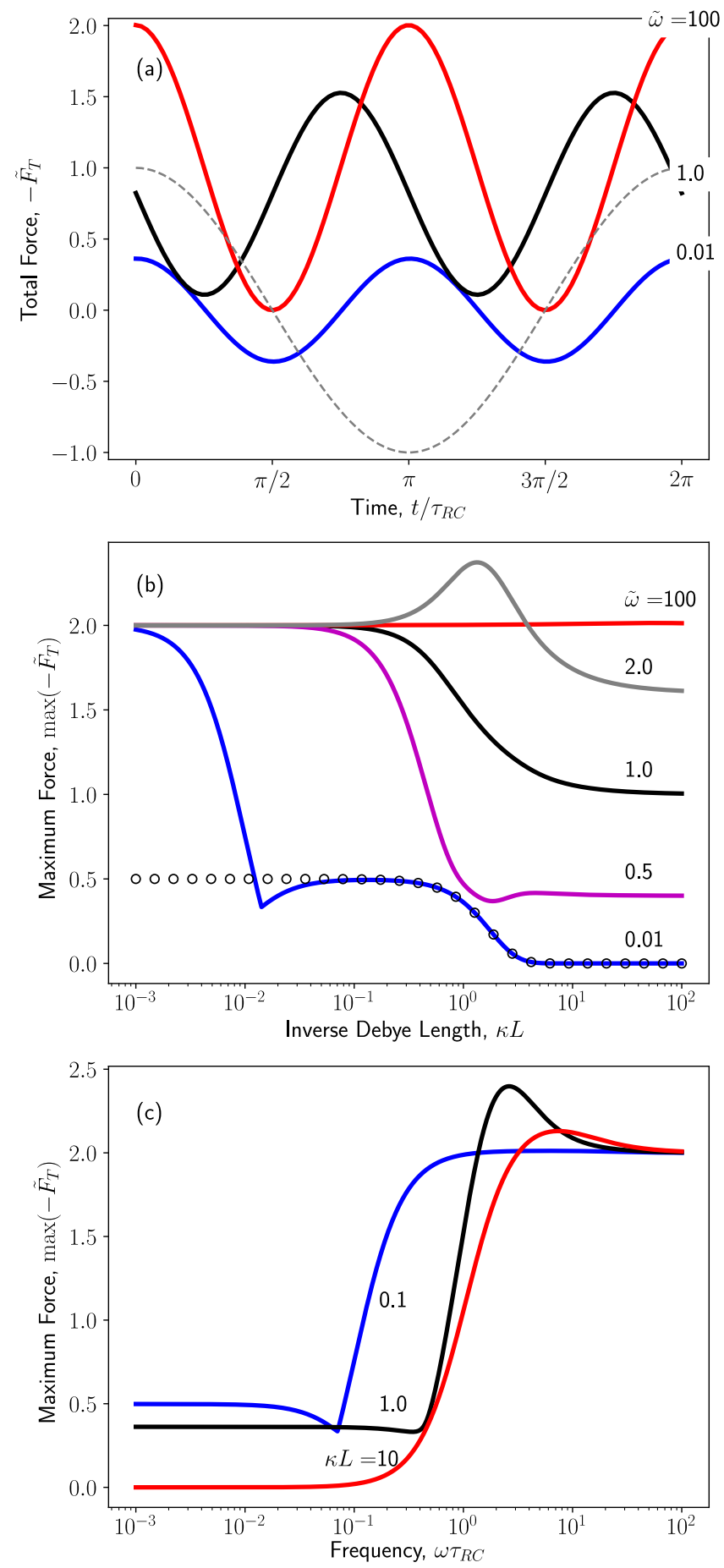

FIG. 2. (a) Total force on the electrode as a function of time over one oscillation cycle, for small, intermediate, and large voltage frequencies $\tilde{\omega}$. The dashed gray curve represents the applied voltage. Here, $\kappa L=1$. (b) The maximum value of force (including steady and oscillating components) over an oscillation cycle as a function of $\kappa L$ for small, intermediate, and large driving frequencies. The symbols represent the quasisteady limit of equilibrium double layer force, and the $\kappa L$ independent force at high frequencies indicates the dielectric limit. (c) The maximum value of force over an oscillation cycle as a function of the driving frequency for three values of $\kappa L$. In all three plots, $D_{+}=D_{-}$and $\tilde{V}_{0}=1$.
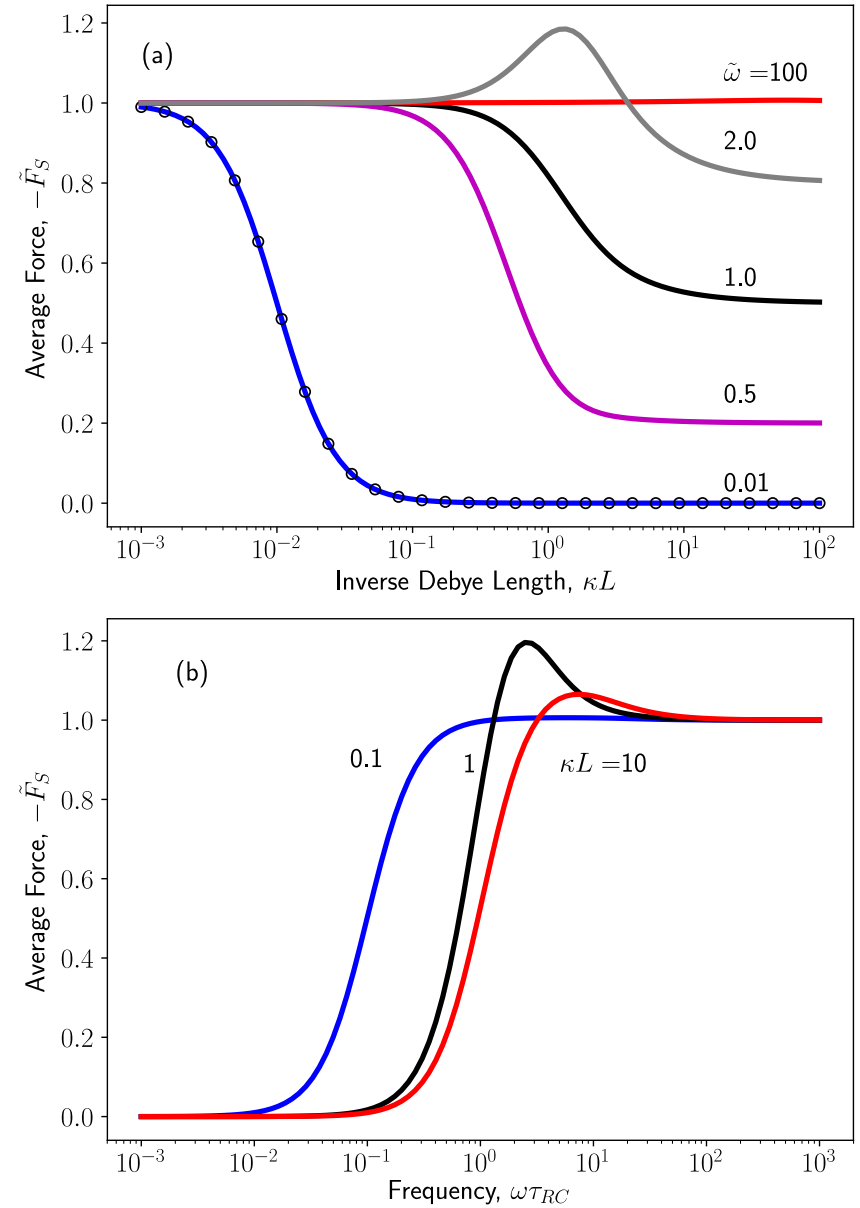

FIG. 3. Steady, time-averaged component of force (a) as a function of $\kappa L$ for low, intermediate, and high driving frequencies. Force is independent of $\kappa L$ at high frequencies, indicating the dielectric limit, and (b) as a function of frequency for three values of $\kappa L$. In (a) the open circles represent the quasisteady limit of the time averaged force. In both plots, $D_{+}=D_{-}$and $\tilde{V}_{0}=1$.

recovers the equilibrium double layer force between surfaces held at constant potentials of equal magnitude and opposite polarity [2]. This is demonstrated in Fig. 2(b) by the open circles (representing magnitude of equilibrium force) overlapping with the blue curve for sufficiently large $\kappa L$ (our dynamic force for $\tilde{\omega} \rightarrow 0$ ). Also in this limit, the time-averaged component $\tilde{F}_{S} \rightarrow-\tilde{\omega}^{2} /\left[\tanh ^{2}(\kappa L)+\tilde{\omega}^{2}\right]$. This is shown in Fig. 3(a) by the open circles (asymptotic expression) overlapping with the blue curve (full expression of steady force). Physically, here the frequency is small compared to the inverse of the double layer charging time. As such, double layers adjacent to each electrode form and relax quasisteadily over an oscillation cycle. Further, for the practically important limit of thin double layers, $\kappa L \gg 1$, the double layers near either surface do not overlap, and the surface potential is almost completely screened. The amplitude of the oscillating force $\left|\tilde{F}_{O}\right| \rightarrow \tilde{V}_{0}^{2} \kappa^{2} L^{2} \exp (-2 \kappa L)$ is exponentially small, and the time-averaged force $\tilde{F}_{S} \rightarrow 0$, as $\kappa L \rightarrow \infty, \tilde{\omega} \rightarrow 0$. Thus, in the quasisteady double layer limit, for the practically relevant 
values of $\kappa L$, the force switches sign within a cycle, indicating a switch between an attraction $\left(\tilde{F}_{T}<0\right)$ and repulsion $\left(\tilde{F}_{T}>0\right)$. This is shown in Fig. 2(a) by the blue curve for $\tilde{\omega}=0.01$.

In the opposite limit of high frequencies, $\tilde{\omega} \rightarrow \infty$, the steady force $\tilde{F}_{S} \rightarrow-\tilde{V}_{0}^{2}$ and the amplitude of oscillations $\left|\tilde{F}_{O}\right| \rightarrow \tilde{V}_{0}^{2}$, which is the force expected from a dielectric capacitor. This is observed in Figs. 2(c) and 3(b) for all values of $\kappa L$ as $\tilde{\omega} \rightarrow \infty$. The total force therefore ranges from $-2 \tilde{V}_{0}^{2}$ to 0 , and is always attractive [red curve for $\tilde{\omega}=100$ in Fig. 2(a)]. Physically, the large frequency does not allow for the formation of double layers; thus the force is independent of $\kappa L$, as is suggested by the red curves for $\tilde{\omega}=100$ in Figs. 2(b) and 3(a). Additionally, as $\kappa L \rightarrow 0$, the force approaches the dielectric limit irrespective of the frequency.

For intermediate frequencies and thin Debye layers $(\kappa L \gg 1)$ the bounds of the force are in between the two limiting cases, as shown by the curves for $\tilde{\omega}=0.5,1$, and 2 in Figs. 2(b) and 3(a). However, for thicker Debye layers $[\kappa L \sim O(1)]$, the maximum and average forces could be greater than their dielectric limits. The instantaneous force switches signs between attractive and repulsive for a small range of frequencies, following which it is always attractive [Fig. 2(a)]. For some frequencies and $\kappa L$, the amplitude of oscillations of the force vanishes; thus the maximum force is just the time-averaged value. This approach to zero appears nonsmooth in Figs. 2(b) and 2(c) as we plot the absolute value of the force. Finally, for intermediate frequencies, the total force undergoes a phase shift [black curve in Fig. 2(a)], relative to either extreme limit of frequency. Indeed it is interesting that the extremes of dielectric and quasisteady response are in phase with one another, despite the entirely different physics.

\section{B. Force chronometry}

We now consider the model cell subject to a suddenly applied voltage. This scenario is relevant in the charging of energy storage devices like supercapacitors. Further, a chronoamperometry experiment [32] measures the current as a function of time, which is analyzed using models relating the current decay to the physicochemical properties of the electrolyte. Below, we suggest that, in addition to the current, one could monitor the force on an electrode to extract the charging time scales and hence the ionic diffusivities and charge carrier concentration.

The dynamics of the force between two charged surfaces under a suddenly applied voltage is governed by the same PNP formulation (1), albeit with a different potential boundary condition, namely, $\tilde{\phi}_{\tilde{x}= \pm 1}= \pm \tilde{V}_{0} \mathcal{H}(\tilde{t})$. We calculate the electric potential in Laplace space which is a function of the normalized Laplace frequency $\tilde{s}$ (scaled by $1 / \tau_{\mathrm{RC}}$ ). This is obtained from the potential in Fourier space by replacing $\iota \tilde{\omega}$ with $\tilde{s}$. The Laplace space potential boundary condition at $\tilde{x}= \pm 1$ is $\tilde{\Phi}= \pm \tilde{V}_{0} \tilde{s}^{-1}$, where $\tilde{\Phi}$ is the normalized potential in Laplace space.

Since the force is quadratic in the potential gradient, the solution for the potential in Laplace space is transformed back to the time domain before calculating the force. The exact expression for the Laplace space potential gradient is given in Appendix B. The inverse Laplace transform is not possible to perform analytically for all times. However, we obtain the asymptotic behavior of the force at long times from the Laplace inverse of the potential gradient in the limit of $\tilde{s} \rightarrow 0$. The long time asymptotic expression for the force is given by (13). To obtain this, we first calculate the pressure distribution from the electric potential by invoking mechanical equilibrium. Similar to the frequency response, the force is independent of position $\tilde{x}$ for the model system considered here. Thus a numerical inverse Laplace transform (using the algorithm from De Hoog et al. [33]) of the $x$-independent part of the potential gradient is used to calculate the dynamics of the force at all times, and is plotted in Fig. 4.

The total (normalized) force on an electrode initially jumps to the dielectric value, $-\tilde{V}_{0}^{2} / 2$ [insets in Figs. 4(a) and 4(c)]. This is expected as the ions are yet to form double layers. This is analogous to the high frequency limit in the spectroscopy analysis. The force decays exponentially at long times, and finally reaches the equilibrium value, $-\frac{1}{4} \tilde{V}_{0}^{2} \kappa^{2} L^{2} \operatorname{cosech}^{2}(\kappa L)$ [inset in Fig. 4(a)], corresponding to fully formed double layers, which is simply the quasisteady limit of our dynamic force spectroscopy. Note that, while all the curves approach the equilibrium value, it is only large enough to distinguish numerically for the smallest $\kappa L$. For this $\kappa L=10$, the total force drops by six orders of magnitude from the initial application of voltage. When unequal diffusivities are considered, we have a dynamic force composed of two distinct decays. Thus, after the initial jump to the dielectric value, we see a second plateau in the force before it approaches its equilibrium value [inset in Fig. 4(c)].

An asymptotic analysis of the Laplace space potential gradient for $\tilde{s} \rightarrow 0$, corresponding to $\tilde{t} \rightarrow \infty$, reveals that the potential, and hence the force, approaches its equilibrium value exponentially. Specifically, the evolution of force is composed of two exponential decays: an initial one with a shorter resistor-capacitor $(\mathrm{RC})$ charging time, $\tilde{\tau}_{\mathrm{RC}}$, corresponding to double layer charging, and a second exponential decay with a longer bulk diffusion time, $\tilde{\tau}_{D}$, due to the difference in ionic diffusivities. The equilibrium force is

$$
\tilde{F}_{\mathrm{eq}}=-\frac{\tilde{V}_{0}^{2}}{4} \kappa^{2} L^{2} \operatorname{cosech}^{2} \kappa L,
$$

and the asymptotic approach to equilibrium,

$$
\tilde{F}_{\text {tot }}-\tilde{F}_{\text {eq }} \equiv \tilde{F}_{\text {dyn }} \sim-\frac{1}{2}\left(\tilde{f}_{\mathrm{RC}} e^{-\tilde{t} / \tilde{\tau}_{\mathrm{RC}}}-\tilde{f}_{D} e^{-\tilde{t} / \tilde{\tau}_{D}}\right)^{2} .
$$

Here the time scales, normalized by the $\mathrm{RC}$ charging time, are

$$
\tilde{\tau}_{\mathrm{RC}}=\frac{D_{A}}{\kappa^{-1} L} \tau_{\mathrm{RC}} \approx \operatorname{coth} \kappa L
$$

and

$$
\tilde{\tau}_{D}=\frac{D_{A}}{\kappa^{-1} L} \tau_{D} \approx \frac{\kappa L}{3}+\operatorname{coth} \kappa L
$$

and the coefficients $\tilde{f}_{\mathrm{RC}}$ and $\tilde{f}_{D}$ are

$$
\tilde{f}_{\mathrm{RC}}=\tilde{V}_{0}, \quad \tilde{f}_{D}=\tilde{V}_{0} \frac{3}{\kappa L}\left(\frac{D_{A}}{D_{F}}\right)^{2} \operatorname{coth} \kappa L .
$$

The RC time is smaller than the bulk diffusion time by a factor of $\kappa L$. However, the coefficient of the diffusive decay 

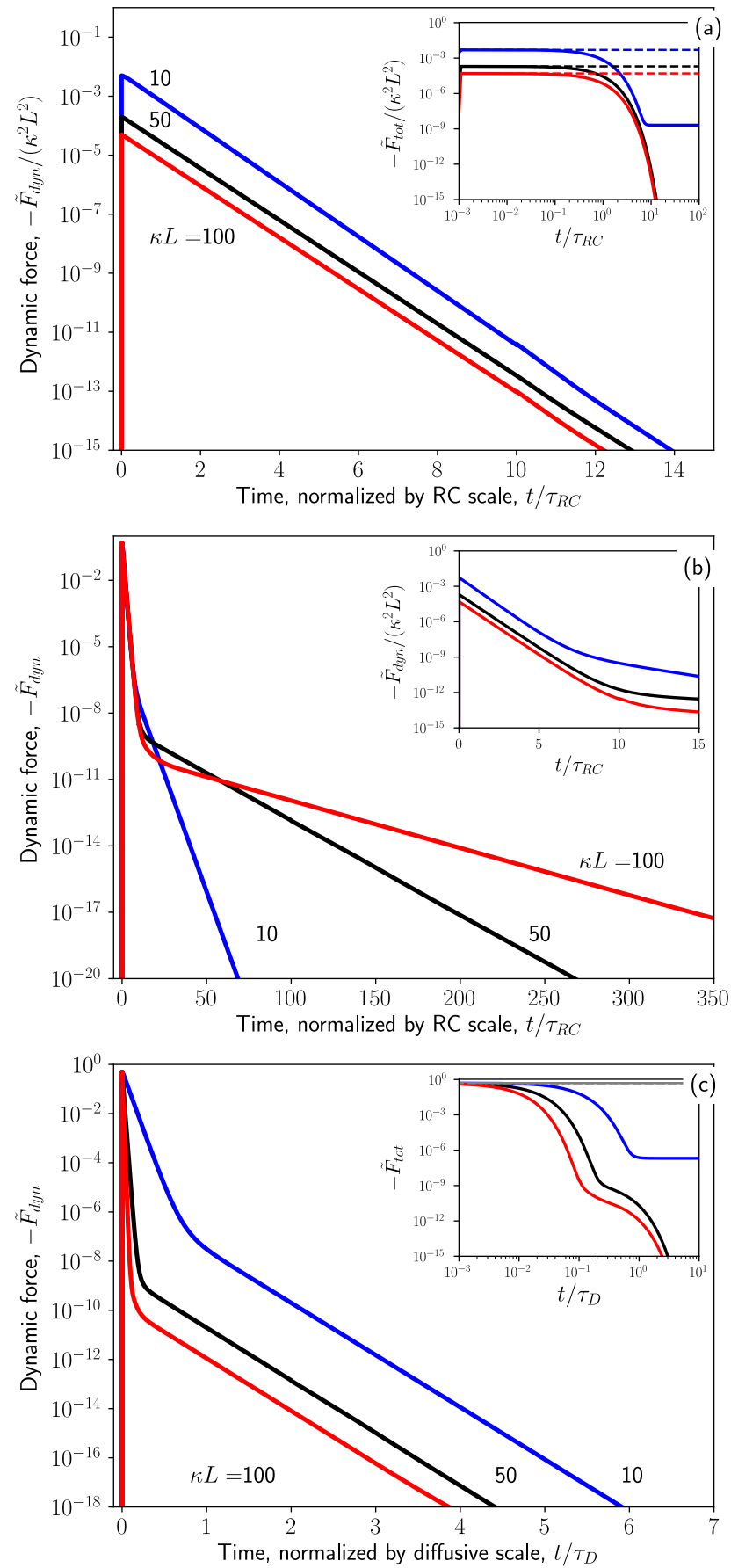

FIG. 4. Deviation from the long-time, equilibrium value of force on the electrode as a function of time for three values of $\kappa L$ for (a) $D_{+}=D_{-}$, where the force exponentially approaches its long time limit on an RC time, $\kappa^{-1} L / D_{A}$. The inset $(\tilde{t}$ scaled logarithmically for clarity) shows the total force going from the dielectric value (dashed lines) to the equilibrium double layer value. Here the force is rescaled by $1 / \kappa^{2} L^{2}$ to separate the three lines which otherwise overlap. (b) $D_{+} \neq D_{-}$, where the initial decay (inset) is on the RC time, followed by a second decay on a bulk diffusion time. (c) $D_{+} \neq$ $D_{-}$, with time rescaled by the diffusive time scale, $L^{2} / D_{A}$. The parallel slopes at longer times indicate a diffusive decay. The inset $(\tilde{t}$ scaled logarithmically) shows the total force undergoing a two-step decay from the dielectric to the equilibrium double layer values. In (b) and (c), $D_{A} / D_{F}=0.18$, corresponding to measurements of ionic diffusivities of sodium and chloride ions in sea water [31]. is smaller than that of the RC decay by a factor of $\kappa L$. Thus, at initial times, one only observes the RC decay, but at longer times, the RC decay becomes subdominant and thus one observes only the diffusive decay. A detailed calculation of the long time behavior of the force from the potential gradient in Laplace space is provided in Appendix B.

In a log-linear plot of $\tilde{F}_{\text {dyn }}$ [Figs. 4(b) and 4(c)] the two exponential decays are represented by straight lines with negative slopes. The initial slope is proportional to the reciprocal of the RC time scale $\left(\tau_{\mathrm{RC}} \sim \kappa^{-1} L / D_{A}\right.$ for $\left.\kappa L \gg 1\right)$, as shown in Fig. 4(b). Hence a measurement of the time dependent force at short times allows one to infer the RC time scale of the system. At long times it depends on the reciprocal of the diffusive time scale $\left(\tau_{D} \sim L^{2} / D_{A}\right.$ for $\kappa L \gg 1$ ), as shown by Fig. 4(c), where the time is scaled by the bulk diffusion time scale. The measurements of the force-time curve at long times can thus be used to infer the ambipolar diffusivity of the salt. The ratio of the RC time and diffusive time scales depends on the Debye length $\left[\tau_{\mathrm{RC}} / \tau_{D} \sim 1 /(\kappa L)\right]$. Thus, from a single force-time curve, one could infer both the ambipolar diffusion coefficient and the Debye length, and therefore the number of charge carriers in the electrolyte.

In the special case of equal ionic diffusivities the long time diffusive time scale disappears and the force reaches its equilibrium value in the order of the RC time [Fig. 4(a)]. This is in agreement with the asymptotic expression where $f_{D}=0$ when $D_{A} / D_{F}=0$ for $D_{+}=D_{-}$. Thus the force evolves solely on the RC time. Considering that all electrolytes have unequal ionic diffusivities on some degree, the bulk diffusion time scale should be expected in general.

\section{Force pulse}

An impulse response measurement under a voltage $\tilde{V}=$ $\tilde{V}_{0} \delta(\tilde{t})$ could also allow one to infer the ambipolar diffusion coefficient and the number of charge carriers. Such a measurement has the advantage of not requiring a sustained applied voltage. We calculate the electric potential gradient in Laplace space and its long time limit from the solution in Fourier space by replacing $\iota \tilde{\omega}$ with $\tilde{s}$. The corresponding (Laplace space) potential boundary condition at $x= \pm 1$ becomes $\tilde{\Phi}=\tilde{V}_{0}$. The long time asymptotic behavior of an impulse voltage is given by $\tilde{F}_{\mathrm{imp}} \sim-\frac{1}{2}\left(\tilde{f}_{\mathrm{RC}} e^{-\tilde{t} / \tilde{\tau}_{\mathrm{RC}}}-\tilde{f}_{D} e^{-\tilde{t} / \tilde{\tau}_{D}}\right)^{2}$. This is the same as the dynamic force obtained for a suddenly applied voltage, with the values of $\tilde{\tau}_{\mathrm{RC}}, \tilde{\tau}_{D}, \tilde{f}_{\mathrm{RC}}$, and $\tilde{f}_{D}$ given by (14), (15), and (16), respectively. Thus the impulse response is also composed of two exponential decays, an initial RC decay followed by a bulk diffusive decay. It differs from the response of the force to a step voltage in two ways: (i) the initial force is infinite as opposed to a finite jump discontinuity and (ii) the force decays to zero at long times, instead of the equilibrium double layer value obtained for a step voltage.

\section{Comparison to experiments}

We now make qualitative comparisons to two recent experimental measurements of the dynamic double layer force. Note that neither of these experiments admit all the simplifying assumptions, namely dilute electrolyte, weak applied 
voltage, and a large separation between electrodes, made by our theory. Hence we cannot make quantitative comparisons.

Surface force measurements performed by Perez-Martinez and Perkin [7] measure the instantaneous time-dependent force between two charged surfaces where the applied voltage oscillates in time. The applied potentials are large $(\approx 7 \mathrm{~V})$ compared to the thermal voltage $(\approx 25 \mathrm{mV})$, and the surfaces are separated by an ionic liquid. They report a nonzero steady state force at long times. While the existence of a steady time averaged force is captured by our theory, the magnitude of the predicted force is significantly different from what they measure. We attribute this to the assumptions of a dilute electrolyte and weak applied voltage, making their experiments very much outside the realm of our calculations.

Measurement of the surface force in response to a suddenly applied voltage has been performed by Tivony et al. [20] in a similar surface force balance. They investigate the charging dynamics of a dilute electrolyte in the case of very narrow electrode separation, mimicking charging in nanopores. The dynamics of force follows a long time exponential decay, which is in agreement with our theory for force chronometry. However, the time scale measured by their experiments is significantly larger than the ones predicted by our theory for the same electrode dimensions and spacing. They attribute the increased time scale to ion migration from the reservoir outside the thin gap between the two charged surfaces in their apparatus. This transport process, therefore, involves ionic fluxes that are not solely normal to the electrode surfaces and is represented as a transmission line model in [20]. We consider a closed, one-dimensional system, where there is no transport of ions beyond the gap between the electrodes. Hence we do not capture this larger time scale.

\section{CONCLUSION}

We developed a theory for the dynamic force between charged surfaces in an electrolyte under a time-dependent voltage. Our work represents a nonequilibrium generalization of the double layer force used in DLVO theory. We envision applications to electrochemical, colloidal, and biological problems in which interactions between bodies or particles with time-dependent surface charges or potentials are encountered. For example, in the directed assembly of colloidal particles under an ac field [34-37], a time-dependent double layer polarization alters the force and, hence, the interaction between the particles. Further, our work can serve as a theoretical foundation for new force-based spectroscopy techniques to analyze electrochemically active surfaces, e.g., during charging or discharging of batteries or supercapacitors. The model problem we have solved could be furthered in a number of interesting directions. Specifically, one could go beyond our analysis using ideal, dilute solution theory in planar geometries under a weak voltage. For instance, steric and electrostatic correlations between ions in concentrated solutions have been extensively studied in the context of charging and discharging dynamics [38-40]; it would be interesting to quantify their impact on dynamic double layer forces. Another useful direction would be to explore the effect of curvature of the electrodes [41], since the surface force apparatus in [7] uses a crossed cylinder geometry. For nonplanar electrodes, one also has to account for the fluid flow and a hydrodynamic pressure along with the osmotic pressure considered here. Recent work regarding rectified electric fields in asymmetric electrolytes under an oscillatory voltage [42] suggests higher order contributions to the force beyond the limit of weak voltages considered here. That is, beyond the weak-voltage limit the frequency response will contain a host of higher harmonics in addition to the zeroth and second mode we consider.

\section{ACKNOWLEDGMENTS}

We acknowledge support from the Camille Dreyfus Teacher-Scholar Award program (A.S.K.) and the Mahmood I. Bhutta Fellowship in Chemical Engineering, Carnegie Mellon University (B.B.)

\section{APPENDIX A: DERIVATION AND SOLUTION OF PNP EQUATIONS UNDER OSCILLATING VOLTAGE}

Here, we derive (1) in the main text. Consider a dilute binary monovalent electrolyte with unequal ionic diffusivities. The ionic fluxes are thus given by

$$
j_{ \pm}=-D_{ \pm}\left(\nabla n_{ \pm} \pm \frac{n_{ \pm} e}{k_{B} T} \nabla \phi\right),
$$

where $n_{ \pm}$is the number concentration of the positive or negative ions, $\phi$ is the electric potential, $k_{B}$ is the Boltzmann constant, $T$ is the temperature, and $e$ is the charge on a proton. The species balances are

$$
\frac{\partial n_{ \pm}}{\partial t}=-\nabla \cdot \boldsymbol{j}_{ \pm} .
$$

For the planar parallel electrodes considered here, the gradients are present only along the direction perpendicular to the electrodes ( $\operatorname{say} x$ ). The ionic species balance equations are thus

$$
\frac{\partial n_{+}}{\partial t}=D_{+}\left[\frac{\partial^{2} n_{+}}{\partial x^{2}}+\frac{\partial}{\partial x}\left(\frac{n_{+} e}{k_{B} T} \frac{\partial \phi}{\partial x}\right)\right]
$$

and

$$
\frac{\partial n_{-}}{\partial t}=D_{-}\left[\frac{\partial^{2} n_{-}}{\partial x^{2}}-\frac{\partial}{\partial x}\left(\frac{n_{-} e}{k_{B} T} \frac{\partial \phi}{\partial x}\right)\right] .
$$

Note that this is the classical PNP model used in previous works $[24,25]$. These equations are linearized in the limit of small applied voltages, $V_{0} /\left(k_{B} T / e\right)=\epsilon \ll 1$, where $k_{B} T / e$ is the thermal voltage, $V_{0}$ is the applied voltage, and $\epsilon$ is a perturbation parameter. Thus the ion concentrations are approximated as a small deviation from their equilibrium value, $n_{ \pm}=n_{\mathrm{eq}}+\epsilon \eta_{ \pm}+O\left(\epsilon^{2}\right)$. The linearized $O(\epsilon)$ equations are, from (A3) and (A4),

$$
\frac{\partial \eta_{+}}{\partial t}=D_{+}\left(\frac{\partial^{2} \eta_{+}}{\partial x^{2}}+\frac{n_{\mathrm{eq}} e}{k_{B} T} \frac{\partial^{2} \phi}{\partial x^{2}}\right)
$$

and

$$
\frac{\partial \eta_{-}}{\partial t}=D_{-}\left(\frac{\partial^{2} \eta_{-}}{\partial x^{2}}-\frac{n_{\mathrm{eq}} e}{k_{B} T} \frac{\partial^{2} \phi}{\partial x^{2}}\right) .
$$

We define the $O(\epsilon)$ charge density, $\rho=e\left(\eta_{+}-\eta_{-}\right)$, and the $O(\epsilon)$ ionic strength, $2 I=\eta_{+}+\eta_{-}$. To reformulate (A5) and 
(A6) in terms of these newly defined quantities, we consider the linear combination $D_{-}$(A5) $\pm D_{+}$(A6) to obtain

$$
D_{-} \frac{\partial \eta_{+}}{\partial t}+D_{+} \frac{\partial \eta_{-}}{\partial t}=2 D_{+} D_{-}\left(\frac{\partial^{2} I}{\partial x^{2}}\right)
$$

and

$$
D_{-} \frac{\partial \eta_{+}}{\partial t}-D_{+} \frac{\partial \eta_{-}}{\partial t}=D_{+} D_{-}\left(\frac{1}{e} \frac{\partial^{2} \rho}{\partial x^{2}}+2 \frac{n_{\mathrm{eq}} e}{k_{B} T} \frac{\partial^{2} \phi}{\partial x^{2}}\right) .
$$

Using $\eta_{+}=I+\rho /(2 e)$ and $\eta_{-}=I-\rho /(2 e)$ in the above equations, and dividing through by $2 D_{+} D_{-}$we get

$$
\frac{\left(D_{-}+D_{+}\right)}{2 D_{+} D_{-}} \frac{\partial I}{\partial t}+\frac{\left(D_{-}-D_{+}\right)}{2 D_{+} D_{-}} \frac{1}{2 e} \frac{\partial \rho}{\partial t}=\frac{\partial^{2} I}{\partial x^{2}}
$$

and

$$
\frac{\left(D_{-}-D_{+}\right)}{2 D_{+} D_{-}} \frac{\partial I}{\partial t}+\frac{\left(D_{-}+D_{+}\right)}{2 D_{+} D_{-}} \frac{1}{2 e} \frac{\partial \rho}{\partial t}=\frac{1}{2 e} \frac{\partial^{2} \rho}{\partial x^{2}}+\frac{n_{\mathrm{eq}} e}{k_{B} T} \frac{\partial^{2} \phi}{\partial x^{2}} .
$$

Defining $D_{A}=2 D_{+} D_{-} /\left(D_{+}+D_{-}\right) \quad$ and $\quad D_{F}=2 D_{+} D_{-} /$ $\left(D_{-}-D_{+}\right)$, we have

$$
\frac{1}{D_{A}} \frac{\partial I}{\partial t}+\frac{1}{D_{F}} \frac{1}{2 e} \frac{\partial \rho}{\partial t}=\frac{\partial^{2} I}{\partial x^{2}}
$$

and

$$
\frac{1}{D_{F}} \frac{\partial I}{\partial t}+\frac{1}{D_{A}} \frac{1}{2 e} \frac{\partial \rho}{\partial t}=\frac{1}{2 e} \frac{\partial^{2} \rho}{\partial x^{2}}+\frac{n_{\mathrm{eq}} e}{k_{B} T} \frac{\partial^{2} \phi}{\partial x^{2}} .
$$

We normalize $I$ with $n_{\text {eq }}, \rho$ with $2 e n_{\text {eq }}, \phi$ with $k_{B} T / e, x$ with $L$, and $t$ with $\tau_{\mathrm{RC}}=\kappa^{-1} L / D_{A}$. The normalized equations are

$$
\kappa L \frac{\partial \tilde{I}}{\partial \tilde{t}}+\kappa L \frac{D_{A}}{D_{F}} \frac{\partial \tilde{\rho}}{\partial \tilde{t}}=\frac{\partial^{2} \tilde{I}}{\partial \tilde{x}^{2}}
$$

and

$$
\kappa L \frac{D_{A}}{D_{F}} \frac{\partial \tilde{I}}{\partial \tilde{t}}+\kappa L \frac{\partial \tilde{\rho}}{\partial \tilde{t}}=\frac{\partial^{2} \tilde{\rho}}{\partial \tilde{x}^{2}}+\frac{\partial^{2} \tilde{\phi}}{\partial \tilde{x}^{2}} .
$$

Finally, $\tilde{\phi}$ is governed by the Poisson equation for electrostatics, whose dimensionless form is given by

$$
\frac{\partial^{2} \tilde{\phi}}{\partial \tilde{x}^{2}}=-\kappa^{2} L^{2} \tilde{\rho} .
$$

Substituting (A15) into the species balances (A13) and (A14), we have

$$
\kappa L \frac{\partial \tilde{I}}{\partial \tilde{t}}+\kappa L \frac{D_{A}}{D_{F}} \frac{\partial \tilde{\rho}}{\partial \tilde{t}}=\frac{\partial^{2} \tilde{I}}{\partial \tilde{x}^{2}}
$$

and

$$
\kappa L \frac{D_{A}}{D_{F}} \frac{\partial \tilde{I}}{\partial \tilde{t}}+\kappa L \frac{\partial \tilde{\rho}}{\partial \tilde{t}}=\frac{\partial^{2} \tilde{\rho}}{\partial \tilde{x}^{2}}-\kappa^{2} L^{2} \tilde{\rho},
$$

which correspond to (1) in the main text. These are solved with the boundary conditions given by (2).

We first consider an oscillating applied voltage $\tilde{V}=$ $\tilde{V}_{0} \cos (\tilde{\omega} \tilde{t})$, where $\tilde{\omega}$ is the angular frequency of oscillations. To solve (A16) and (A17), we recognize that these are coupled linear, second order partial differential equations. The Fourier transform of (A16) and (A17), defined for this case of a single oscillating frequency as $\tilde{g}=2 \operatorname{Re}\left(\tilde{g}_{1} e^{i \tilde{\omega} \tilde{t}}\right)$, where $\tilde{g}_{1}$ is a complex valued function of $\tilde{x}$ and $\tilde{\omega}, \iota=\sqrt{-1}, \operatorname{Re}$ is for the real part, and $\tilde{g}$ represents $\tilde{\rho}, \tilde{I}$, or $\tilde{\phi}$, yields a set of ordinary differential equations independent of time given by

$$
\begin{aligned}
\frac{\partial^{2} \tilde{\rho}_{1}}{\partial \tilde{x}^{2}} & =\iota \tilde{\omega} \kappa L \tilde{\rho}_{1}+\kappa^{2} L^{2} \tilde{\rho}_{1}+\iota \tilde{\omega} \kappa L \frac{D_{A}}{D_{F}} \tilde{I}_{1}, \\
\frac{\partial^{2} \tilde{I}_{1}}{\partial \tilde{x}^{2}} & =\iota \tilde{\omega} \kappa L \tilde{I}_{1}+\iota \tilde{\omega} \kappa L \frac{D_{A}}{D_{F}} \tilde{\rho}_{1} .
\end{aligned}
$$

The corresponding potential and flux boundary conditions in Fourier space are

$$
\left.\tilde{\phi}_{1}\right|_{ \pm 1}= \pm \tilde{V}_{0}
$$

and

$$
\left(\frac{\partial \tilde{\rho}}{\partial \tilde{x}}+\frac{\partial \tilde{\phi}}{\partial \tilde{x}}\right)_{ \pm 1}=0, \quad\left(\frac{\partial \tilde{I}}{\partial \tilde{x}}\right)_{ \pm 1}=0 .
$$

The linearized ordinary differential equations can be written as

$$
\frac{d^{2}}{d \tilde{x}^{2}}\left(\begin{array}{c}
\tilde{\rho}_{1} \\
\tilde{I}_{1}
\end{array}\right)=\left(\begin{array}{cc}
\kappa^{2} L^{2}+\iota \tilde{\omega} \kappa L & \iota \tilde{\omega} \kappa L \frac{D_{A}}{D_{F}} \\
\iota \tilde{\omega} \kappa L \frac{D_{A}}{D_{F}} & \iota \tilde{\omega} \kappa L
\end{array}\right)\left(\begin{array}{l}
\tilde{\rho}_{1} \\
\tilde{I}_{1}
\end{array}\right) .
$$

This is of the form $\boldsymbol{u}^{\prime \prime}=\boldsymbol{A} \boldsymbol{u}$. To decouple the two equations, we perform an eigenvalue decomposition of the matrix $\boldsymbol{A}$. That is, $\boldsymbol{A}=\boldsymbol{P} \boldsymbol{D} \boldsymbol{P}^{-1}$, where the eigenvalues of $\boldsymbol{A}$ form the elements of the diagonal matrix $\boldsymbol{D}$, and the corresponding eigenvectors form the columns of $\boldsymbol{P}$ and $\boldsymbol{P}^{-1}$ is the inverse of $\boldsymbol{P}$. Thus we have $\boldsymbol{u}^{\prime \prime}=\boldsymbol{P D P} \boldsymbol{P}^{-1} \boldsymbol{u}$. Defining a transformed variable vector, $\boldsymbol{y}=\boldsymbol{P}^{-1} \boldsymbol{u}$, we get $\boldsymbol{y}^{\prime \prime}=\boldsymbol{D} \boldsymbol{y}$. Since $\boldsymbol{D}$ is a diagonal matrix, this represents a decoupled set of equations that are readily solved. The solution in terms of the original variables is then obtained using the inverse transformation, $\boldsymbol{P} \boldsymbol{y}=\boldsymbol{u}$. The eigenvalues and eigenvectors of $\boldsymbol{A}$ are obtained using

$$
\lambda_{i}^{2}=\frac{\operatorname{Tr}(\boldsymbol{A}) \pm \sqrt{[\operatorname{Tr}(\boldsymbol{A})]^{2}-4[\operatorname{Det}(\boldsymbol{A})]}}{2}
$$

and the corresponding eigenvectors, $\boldsymbol{v}_{i}$, are

$$
\boldsymbol{v}_{i}=\left(\begin{array}{c}
\frac{A_{11}-A_{22} \pm \sqrt{[\operatorname{Tr}(\boldsymbol{A})]^{2}-4[\operatorname{Det}(A)]}}{2 A_{12}} \\
1
\end{array}\right) .
$$

The eigenvalues and eigenvectors are [as given in (7) and (8) of the main text]

$$
\lambda_{i}^{2}=\frac{\kappa^{2} L^{2}}{2}+\iota \tilde{\omega} \kappa L \pm \frac{\kappa^{2} L^{2}}{2} \sqrt{\left(1-\frac{4 \tilde{\omega}^{2}}{\kappa^{2} L^{2}} \frac{D_{A}^{2}}{D_{F}^{2}}\right)}
$$

and

$$
\frac{v_{2}}{v_{1}}=\frac{1-\sqrt{\left(1-\frac{4 \tilde{\omega}^{2}}{\kappa^{2} L^{2}} \frac{D_{A}^{2}}{D_{F}^{2}}\right)}}{1+\sqrt{\left(1-\frac{4 \tilde{\omega}^{2}}{\kappa^{2} L^{2}} \frac{D_{A}^{2}}{D_{F}^{2}}\right)}} .
$$

The solution in terms of the transformed variables $y$ is

$$
\begin{aligned}
& y_{1}=a_{1} \sinh \left(\lambda_{1} \tilde{x}\right), \\
& y_{2}=a_{2} \sinh \left(\lambda_{2} \tilde{x}\right) .
\end{aligned}
$$


Here, we have invoked antisymmetry due to the applied voltage being an odd function about the center of the cell. Transforming back to the original variables gives

$$
\begin{aligned}
& u_{1}=\tilde{\rho}_{1}=v_{1} a_{1} \sinh \left(\lambda_{1} \tilde{x}\right)+v_{2} a_{2} \sinh \left(\lambda_{2} \tilde{x}\right), \\
& u_{2}=\tilde{I}_{1}=a_{1} \sinh \left(\lambda_{1} \tilde{x}\right)+a_{2} \sinh \left(\lambda_{2} \tilde{x}\right) .
\end{aligned}
$$

The potential and potential gradient are calculated from the above solution using (A15) as

$$
\frac{\partial \tilde{\phi}_{1}}{\partial \tilde{x}}=-\kappa^{2} L^{2}\left(\frac{v_{1} a_{1}}{\lambda_{1}} \cosh \left(\lambda_{1} \tilde{x}\right)+\frac{v_{2} a_{2}}{\lambda_{2}} \cosh \left(\lambda_{2} \tilde{x}\right)\right)+c_{1}
$$

and

$\tilde{\phi}_{1}=-\kappa^{2} L^{2}\left(\frac{v_{1} a_{1}}{\lambda_{1}^{2}} \sinh \left(\lambda_{1} \tilde{x}\right)+\frac{v_{2} a_{2}}{\lambda_{2}^{2}} \sinh \left(\lambda_{2} \tilde{x}\right)\right)+c_{1} \tilde{x}+c_{2}$.

The values of $a_{1}, a_{2}, c_{1}$, and $c_{2}$ are found using the boundary conditions (A19) and (A20).

\section{APPENDIX B: CALCULATION OF THE DYNAMIC DOUBLE LAYER PRESSURE}

The pressure $\tilde{p}$ is calculated from the solution for the electric field by invoking mechanical equilibrium

$$
\nabla \tilde{p}+\nabla \cdot \tilde{\boldsymbol{\sigma}}_{E}=0,
$$

where $\sigma_{E}$ is the electric stress tensor, defined by $\sigma_{E}=$ $\varepsilon\left(\nabla \phi \nabla \phi-\frac{1}{2}(\nabla \phi) \cdot(\nabla \phi) \boldsymbol{I}\right)$, where $\boldsymbol{I}$ is the identity matrix. For the one dimensional system considered here, the mechanical equilibrium reduces to

$$
\frac{\partial \tilde{p}}{\partial \tilde{x}}=\frac{\partial}{\partial \tilde{x}}\left[\frac{1}{2}\left(\frac{\partial \tilde{\phi}}{\partial \tilde{x}}\right)^{2}\right]
$$

From the solution of the PNP equations, we have the potential gradient in Fourier space [as (5) in main text]

$$
\begin{aligned}
\frac{\partial \tilde{\phi}_{1}}{\partial \tilde{x}}=\frac{\tilde{V}_{0}}{Q} & {\left[-\kappa^{2} L^{2}\left(\frac{\cosh \left(\lambda_{1} \tilde{x}\right)}{\lambda_{1} \cosh \left(\lambda_{1}\right)}-\frac{\lambda_{1}}{\lambda_{2}} \frac{v_{2}}{v_{1}} \frac{\cosh \left(\lambda_{2} \tilde{x}\right)}{\lambda_{2} \cosh \left(\lambda_{2}\right)}\right)\right.} \\
& \left.+\left(\lambda_{1}-\frac{\kappa^{2} L^{2}}{\lambda_{1}}\right)-\frac{\lambda_{1}}{\lambda_{2}} \frac{v_{2}}{v_{1}}\left(\lambda_{2}-\frac{\kappa^{2} L^{2}}{\lambda_{2}}\right)\right] .
\end{aligned}
$$

Here,

$$
\begin{aligned}
Q= & {\left[\left(\lambda_{1}-\frac{\kappa^{2} L^{2}}{\lambda_{1}}\right)-\frac{\lambda_{1}}{\lambda_{2}} \frac{v_{2}}{v_{1}}\left(\lambda_{2}-\frac{\kappa^{2} L^{2}}{\lambda_{2}}\right)\right.} \\
& \left.+\frac{\kappa^{2} L^{2}}{\lambda_{1}^{2}} \tanh \left(\lambda_{1}\right)-\frac{\lambda_{1}}{\lambda_{2}} \frac{v_{2}}{v_{1}} \frac{\kappa^{2} L^{2}}{\lambda_{2}^{2}} \tanh \left(\lambda_{2}\right)\right] .
\end{aligned}
$$

The potential gradient can be expressed as

$$
\frac{\partial \tilde{\phi}_{1}}{\partial \tilde{x}}=\alpha_{1} \cosh \left(\lambda_{1} \tilde{x}\right)+\alpha_{2} \cosh \left(\lambda_{2} \tilde{x}\right)+\beta,
$$

where

$$
\begin{aligned}
\alpha_{1} & =\frac{-\kappa^{2} L^{2} \tilde{V}_{0}}{\lambda_{1} \cosh \left(\lambda_{1}\right) Q}, \\
\alpha_{2} & =\frac{\lambda_{1} v_{2}}{\lambda_{2} v_{1}} \frac{\kappa^{2} L^{2} \tilde{V}_{0}}{\lambda_{2} \cosh \left(\lambda_{2}\right) Q},
\end{aligned}
$$

and

$$
\beta=\frac{\tilde{V}_{0}}{Q}\left[\left(\lambda_{1}-\frac{\kappa^{2} L^{2}}{\lambda_{1}}\right)-\frac{\lambda_{1}}{\lambda_{2}} \frac{v_{2}}{v_{1}}\left(\lambda_{2}-\frac{\kappa^{2} L^{2}}{\lambda_{2}}\right)\right] .
$$

Since the pressure calculation involves the square of the potential gradient, we have to convert from Fourier space to the time domain. For a single frequency response we have

$$
\frac{\partial \tilde{\phi}}{\partial \tilde{x}}=2 \operatorname{Re}\left(\frac{\partial \tilde{\phi}_{1}}{\partial \tilde{x}} e^{\imath \omega \tilde{t}}\right)=\left(\frac{\partial \tilde{\phi}_{1}}{\partial \tilde{x}} e^{i \tilde{\omega} \tilde{t}}+\frac{\partial \tilde{\phi}_{1}^{*}}{\partial \tilde{x}} e^{-i \tilde{\omega} \tilde{t}}\right),
$$

where $\tilde{\phi}_{1}^{*}$ is the complex conjugate of $\tilde{\phi}_{1}$. The square of the potential gradient is then

$$
\begin{aligned}
& \left(\frac{\partial \tilde{\phi}}{\partial \tilde{x}}\right)^{2}=\left(\frac{\partial \tilde{\phi}_{1}}{\partial \tilde{x}} e^{i \tilde{\omega} \tilde{t}}+\frac{\partial \tilde{\phi}_{1}^{*}}{\partial \tilde{x}} e^{-i \tilde{\omega} \tilde{t}}\right)^{2} \\
& =\left\{\left[\alpha_{1} \cosh \left(\lambda_{1} \tilde{x}\right)+\alpha_{2} \cosh \left(\lambda_{2} \tilde{x}\right)+\beta\right] e^{i \tilde{\omega} \tilde{t}}+\left[\alpha_{1}^{*} \cosh \left(\lambda_{1}^{*} \tilde{x}\right)+\alpha_{2}^{*} \cosh \left(\lambda_{2}^{*} \tilde{x}\right)+\beta^{*}\right] e^{-l \tilde{\omega} \tilde{t}}\right\}^{2} \\
& =\left[\alpha_{1} \cosh \left(\lambda_{1} \tilde{x}\right)+\alpha_{2} \cosh \left(\lambda_{2} \tilde{x}\right)+\beta\right]^{2} e^{2 \imath \tilde{\omega} \tilde{t}}+\left[\alpha_{1}^{*} \cosh \left(\lambda_{1}^{*} \tilde{x}\right)+\alpha_{2}^{*} \cosh \left(\lambda_{2}^{*} \tilde{x}\right)+\beta^{*}\right]^{2} e^{-2 \imath \tilde{\omega} \tilde{t}} \\
& +2\left\{\left[\alpha_{1} \cosh \left(\lambda_{1} \tilde{x}\right)+\alpha_{2} \cosh \left(\lambda_{2} \tilde{x}\right)+\beta\right]\left[\alpha_{1}^{*} \cosh \left(\lambda_{1}^{*} \tilde{x}\right)+\alpha_{2}^{*} \cosh \left(\lambda_{2}^{*} \tilde{x}\right)+\beta^{*}\right]\right\} \\
& =2 \operatorname{Re}\left(\left[\frac{\alpha_{1}^{2}}{2}+\frac{\alpha_{2}^{2}}{2}+\beta^{2}+\frac{\alpha_{1}^{2} \cosh \left(2 \lambda_{1} \tilde{x}\right)}{2}+\frac{\alpha_{2}^{2} \cosh \left(2 \lambda_{2} \tilde{x}\right)}{2}+2 \alpha_{1} \alpha_{2} \cosh \left(\lambda_{1} \tilde{x}\right) \cosh \left(\lambda_{2} \tilde{x}\right)\right.\right. \\
& \left.\left.+2 \alpha_{1} \beta \cosh \left(\lambda_{1} \tilde{x}\right)+2 \alpha_{2} \beta \cosh \left(\lambda_{2} \tilde{x}\right)\right] e^{2 l \tilde{\omega} \tilde{t}}\right)+2\left[\alpha_{1} \alpha_{1}^{*} \cosh \left(\lambda_{1} \tilde{x}\right) \cosh \left(\lambda_{1}^{*} \tilde{x}\right)+\alpha_{2} \alpha_{2}^{*} \cosh \left(\lambda_{2} \tilde{x}\right) \cosh \left(\lambda_{2}^{*} \tilde{x}\right)\right. \\
& +\alpha_{1} \alpha_{2}^{*} \cosh \left(\lambda_{1} \tilde{x}\right) \cosh \left(\lambda_{2}^{*} \tilde{x}\right)+\alpha_{2} \alpha_{1}^{*} \cosh \left(\lambda_{2} \tilde{x}\right) \cosh \left(\lambda_{1}^{*} \tilde{x}\right)+\alpha_{1}^{*} \cosh \left(\lambda_{1}^{*} \tilde{x}\right) \beta+\alpha_{1} \cosh \left(\lambda_{1} \tilde{x}\right) \beta^{*} \\
& \left.+\alpha_{2} \cosh \left(\lambda_{2} \tilde{x}\right) \beta+\alpha_{2}^{*} \cosh \left(\lambda_{2}^{*} \tilde{x}\right) \beta+\beta \beta^{*}\right] \text {. }
\end{aligned}
$$

This can be written, for brevity, as

$$
\left(\frac{\partial \tilde{\phi}}{\partial \tilde{x}}\right)^{2}=2 \operatorname{Re}\left[\left(\frac{\alpha_{1}^{2}}{2}+\frac{\alpha_{2}^{2}}{2}+\beta^{2}+\gamma(\tilde{x})\right) e^{2 i \tilde{\omega} \tilde{t}}\right]+2\left[\beta \beta^{*}+v(\tilde{x})\right],
$$


where

$$
\begin{aligned}
\gamma(\tilde{x})= & \frac{\alpha_{1}^{2} \cosh \left(2 \lambda_{1} \tilde{x}\right)}{2}+\frac{\alpha_{2}^{2} \cosh \left(2 \lambda_{2} \tilde{x}\right)}{2} \\
& +2 \alpha_{1} \alpha_{2} \cosh \left(\lambda_{1} \tilde{x}\right) \cosh \left(\lambda_{2} \tilde{x}\right)+2 \alpha_{1} \beta \cosh \left(\lambda_{1} \tilde{x}\right) \\
& +2 \alpha_{2} \beta \cosh \left(\lambda_{2} \tilde{x}\right)
\end{aligned}
$$

and

$$
\begin{aligned}
v(\tilde{x})= & {\left[\alpha_{1} \alpha_{1}^{*} \cosh \left(\lambda_{1} \tilde{x}\right) \cosh \left(\lambda_{1}^{*} \tilde{x}\right)\right.} \\
& +\alpha_{2} \alpha_{2}^{*} \cosh \left(\lambda_{2} \tilde{x}\right) \cosh \left(\lambda_{2}^{*} \tilde{x}\right) \\
& +\alpha_{1} \alpha_{2}^{*} \cosh \left(\lambda_{1} \tilde{x}\right) \cosh \left(\lambda_{2}^{*} \tilde{x}\right) \\
& +\alpha_{2} \alpha_{1}^{*} \cosh \left(\lambda_{2} \tilde{x}\right) \cosh \left(\lambda_{1}^{*} \tilde{x}\right) \\
& +\alpha_{1}^{*} \cosh \left(\lambda_{1}^{*} \tilde{x}\right) \beta+\alpha_{1} \cosh \left(\lambda_{1} \tilde{x}\right) \beta^{*} \\
& \left.+\alpha_{2} \cosh \left(\lambda_{2} \tilde{x}\right) \beta+\alpha_{2}^{*} \cosh \left(\lambda_{2}^{*} \tilde{x}\right) \beta\right] .
\end{aligned}
$$

We choose to express the square of the potential gradient in this way, separating the $x$-dependent and $x$-independent parts to clarify the integration and differentiation in the next steps. The mechanical equilibrium (B2) gives

$$
\begin{gathered}
\tilde{p}=\int_{\tilde{x}}\left(\frac { \partial } { \partial \tilde { x } } \left\{\operatorname{Re}\left[\left(\frac{\alpha_{1}^{2}}{2}+\frac{\alpha_{2}^{2}}{2}+\beta^{2}+\gamma(\tilde{x})\right) e^{2 i \tilde{\omega} \tilde{t}}\right]\right.\right. \\
\left.\left.+\left[\beta \beta^{*}+v(\tilde{x})\right]\right\}\right) d \tilde{x} \\
=\int_{\tilde{x}}\left(\operatorname{Re}\left[\frac{d \gamma}{\tilde{x}} e^{2 i \tilde{\omega} \tilde{t}}\right]+\frac{d \nu}{d \tilde{x}}\right) d \tilde{x}=\operatorname{Re}\left[\gamma(\tilde{x}) e^{2 i \tilde{\omega} \tilde{t}}\right]+v(\tilde{x})+\mathrm{c} .
\end{gathered}
$$

Here $c$ is an integration constant, which we identify as a "reservoir" pressure, $\tilde{p}_{0}$. This is equivalently the osmotic pressure in the electrolyte when the surface is uncharged. In this limit, $\gamma(\tilde{x})=0$ and $v(\tilde{x})=0$. Thus

$$
\tilde{p}-\tilde{p}_{0}=\operatorname{Re}\left[\gamma(\tilde{x}) e^{2 l \tilde{\omega} \tilde{t}}\right]+v(\tilde{x}) .
$$

The total force per unit area on the electrode is given by

$$
\tilde{F}_{T}=\tilde{p}-\tilde{p}_{0}-\frac{1}{2}\left(\frac{\partial \tilde{\phi}}{\partial \tilde{x}}\right)^{2} .
$$

Using (B11) and (B15) yields

$$
\tilde{F}_{T}=-\operatorname{Re}\left[\left(\frac{\alpha_{1}^{2}}{2}+\frac{\alpha_{2}^{2}}{2}+\beta^{2}\right) e^{2 \imath \tilde{\omega} \tilde{t}}\right]-\left[\beta \beta^{*}\right],
$$

where the first term represents the oscillating part of the force and the second term is the steady, time-averaged value. In terms of the notations used in the main text, these are

$$
\tilde{F}_{O}=-\frac{\alpha_{1}^{2}}{2}-\frac{\alpha_{2}^{2}}{2}-\beta^{2}, \quad \tilde{F}_{S}=-\beta \beta^{*}=-|\beta|^{2} .
$$

Substituting for $\alpha_{1}, \alpha_{2}$, and $\beta$, we have the expressions for the force as given in the main text; namely (10) and (11).

To obtain the step response of the force, the potential gradient in Laplace space $(\partial \tilde{\Phi} / \partial \tilde{x})$ is obtained from that in the Fourier space $\left(\partial \tilde{\phi}_{1} / \partial \tilde{x}\right)$ by substituting the frequency $\iota \tilde{\omega}$ with the Laplace variable $\tilde{s}$. Thus

$$
\begin{aligned}
\frac{\partial \tilde{\Phi}}{\partial \tilde{x}}=\frac{\tilde{V}_{0}}{Q^{s}} & {\left[-\kappa^{2} L^{2}\left(\frac{\cosh \left(\lambda_{1}^{s} \tilde{x}\right)}{\lambda_{1}^{s} \cosh \left(\lambda_{1}^{s}\right)}-\frac{\lambda_{1}^{s}}{\lambda_{2}^{s}} \frac{v_{2}^{s}}{v_{1}^{s}} \frac{\cosh \left(\lambda_{2}^{s} \tilde{x}\right)}{\lambda_{2}^{s} \cosh \left(\lambda_{2}^{s}\right)}\right)\right.} \\
& \left.+\left(\lambda_{1}^{s}-\frac{\kappa^{2} L^{2}}{\lambda_{1}^{s}}\right)-\frac{\lambda_{1}^{s}}{\lambda_{2}^{s}} \frac{v_{2}^{s}}{v_{1}^{s}}\left(\lambda_{2}^{s}-\frac{\kappa^{2} L^{2}}{\lambda_{2}^{s}}\right)\right] .
\end{aligned}
$$

Here,

$$
\begin{aligned}
Q= & {\left[\left(\lambda_{1}^{s}-\frac{\kappa^{2} L^{2}}{\lambda_{1}^{s}}\right)-\frac{\lambda_{1}^{s}}{\lambda_{2}^{s}} \frac{v_{2}^{s}}{v_{1}^{s}}\left(\lambda_{2}^{s}-\frac{\kappa^{2} L^{2}}{\lambda_{2}^{s}}\right)\right.} \\
& \left.+\frac{\kappa^{2} L^{2}}{\left(\lambda_{1}^{2}\right)^{s}} \tanh \lambda_{1}^{s}-\frac{\lambda_{1}^{s}}{\lambda_{2}^{s}} \frac{v_{2}^{s}}{v_{1}^{s}} \frac{\kappa^{2} L^{2}}{\left(\lambda_{2}^{s}\right)^{2}} \tanh \lambda_{2}^{s}\right],
\end{aligned}
$$

$\lambda_{1}^{s}$ and $\lambda_{2}^{s}$ are the eigenvalues of the system of ODEs governing charge density and ionic strength in Laplace space, and $\left[v_{1}^{s}, 1\right]$ and $\left[v_{2}^{s}, 1\right]$ are the corresponding eigenvectors. Thus

$$
\left(\lambda_{i}^{s}\right)^{2}=\frac{\kappa^{2} L^{2}}{2}+\tilde{s} \kappa L \pm \frac{\kappa^{2} L^{2}}{2} \sqrt{\left(1+\frac{4 \tilde{s}^{2}}{\kappa^{2} L^{2}} \frac{D_{A}^{2}}{D_{F}^{2}}\right)}
$$

and

$$
\frac{v_{2}^{s}}{v_{1}^{s}}=\frac{1-\sqrt{\left(1+\frac{4 \tilde{s}^{2}}{\kappa^{2} L^{2}} \frac{D_{A}^{2}}{D_{F}^{2}}\right)}}{1+\sqrt{\left(1+\frac{4 \tilde{S}^{2}}{\kappa^{2} L^{2}} \frac{D_{A}^{2}}{D_{F}^{2}}\right)}},
$$

where $\tilde{s}=s D_{A} \kappa / L$ is the normalized Laplace variable. The electric potential gradient in Laplace space can be expressed as

$$
\frac{\partial \tilde{\Phi}}{\partial \tilde{x}}=\alpha_{1}^{s} \cosh \left(\lambda_{1}^{s} \tilde{x}\right)+\alpha_{2}^{s} \cosh \left(\lambda_{2}^{s} \tilde{x}\right)+\beta^{s},
$$

where

$$
\begin{aligned}
& \alpha_{1}^{s}=\frac{-\kappa^{2} L^{2} \tilde{V}_{0}}{\lambda_{1}^{s} \cosh \left(\lambda_{1}^{s}\right) Q^{s},} \\
& \alpha_{2}^{s}=\frac{\lambda_{1}^{s} v_{2}^{s}}{\lambda_{2}^{s} v_{1}^{s}} \frac{\kappa^{2} L^{2} \tilde{V}_{0}}{\lambda_{2}^{s} \cosh \left(\lambda_{2}^{s}\right) Q^{s}},
\end{aligned}
$$

and

$$
\beta=\frac{\tilde{V}_{0}}{Q^{s}}\left[\left(\lambda_{1}^{s}-\frac{\kappa^{2} L^{2}}{\lambda_{1}^{s}}\right)-\frac{\lambda_{1}^{s}}{\lambda_{2}^{s}} \frac{v_{2}^{s}}{v_{1}^{s}}\left(\lambda_{2}^{s}-\frac{\kappa^{2} L^{2}}{\lambda_{2}^{s}}\right)\right] .
$$

To obtain the force, we first invert this back to the time domain. This is not possible to do in closed form; however, we obtain an asymptotic behavior at long times by taking the limit of the potential gradient in Laplace space as the Laplace variable $\tilde{s} \rightarrow 0$, and then inverting it to the time domain. This gives

$$
\begin{aligned}
\lim _{\tilde{s} \rightarrow 0} \frac{\partial \tilde{\Phi}}{\partial \tilde{x}}= & \frac{f_{x} \cosh (\kappa L \tilde{x})}{\tilde{s}}-\frac{f_{x} \cosh (\kappa L \tilde{x})}{\tilde{s}+1 / \tilde{\tau}_{x}} \\
& +\frac{f_{\mathrm{RC}}}{\tilde{s}+1 / \tilde{\tau}_{\mathrm{RC}}}+\frac{f_{D}}{\tilde{s}+1 / \tilde{\tau}_{D}},
\end{aligned}
$$

where

$$
\begin{aligned}
f_{x} & =\tilde{V}_{0} \kappa L \operatorname{cosech}(\kappa L), \\
f_{\mathrm{RC}} & =\tilde{V}_{0},
\end{aligned}
$$


and

$$
f_{D}=-\tilde{V}_{0} \frac{3}{\kappa L}\left(\frac{D_{A}}{D_{F}}\right)^{2} \operatorname{coth}(\kappa L) .
$$

Here, $f_{x}, f_{\mathrm{RC}}$, and $f_{D}$ are independent of $x$. The time scales (normalized by the RC time $\frac{D_{A}}{\kappa^{-1} L}$ ) are

$$
\begin{aligned}
\tilde{\tau}_{x} & =\operatorname{coth}(\kappa L)+\frac{\tanh (\kappa L)}{2}-\frac{\tanh (\kappa L \tilde{x})}{2}+O\left(\frac{1}{\kappa L}\right), \\
\tilde{\tau}_{\mathrm{RC}} & =\operatorname{coth}(\kappa L)+O\left(\frac{1}{\kappa L}\right),
\end{aligned}
$$

and

$$
\tilde{\tau}_{D}=\frac{\kappa L}{3}+\operatorname{coth}(\kappa L)+O\left(\frac{1}{\kappa L}\right) .
$$

The inverse Laplace transform of (B27) thus gives the long-time behavior

$$
\frac{\partial \tilde{\phi}}{\partial \tilde{x}} \sim \cosh (\kappa L x)\left(f_{x}-f_{x} e^{-\tilde{t} / \tilde{\tau}_{x}}\right)+f_{\mathrm{RC}} e^{-\tilde{t} / \tilde{\tau}_{\mathrm{RC}}}+f_{D} e^{-\tilde{t} / \tilde{\tau}_{D}} .
$$

The long-time pressure gradient is again calculated using mechanical equilibrium (B2)

$$
\frac{\partial \tilde{p}}{\partial \tilde{x}} \sim \int_{\tilde{x}} \frac{1}{2} \frac{\partial}{\partial \tilde{x}}\left[\cosh (\kappa L x)\left(f_{x}-f_{x} e^{-\tilde{t} / \tilde{\tau}_{x}}\right)+f_{\mathrm{RC}} e^{-\tilde{t} / \tilde{\tau}_{\mathrm{RC}}}+f_{D} e^{-\tilde{t} / \tilde{\tau}_{D}}\right]^{2} d \tilde{x} .
$$

Thus

$$
\begin{aligned}
\frac{\partial \tilde{p}}{\partial \tilde{x}}=\int_{\tilde{x}} \frac{1}{2} \frac{\partial}{\partial \tilde{x}} & {\left[\frac{1+\cosh 2 \kappa L}{2}\left(f_{x}-f_{x} e^{-\tilde{t} / \tilde{\tau}_{x}}\right)^{2}+\left(f_{\mathrm{RC}} e^{-\tilde{t} / \tilde{\tau}_{\mathrm{RC}}}+f_{D} e^{-\tilde{t} / \tilde{\tau}_{D}}\right)^{2}\right.} \\
& \left.+2\left\{\cosh (\kappa L x)\left(f_{x}-f_{x} e^{-\tilde{t} / \tilde{\tau}_{x}}\right)\left(f_{\mathrm{RC}} e^{-\tilde{t} / \tilde{\tau}_{\mathrm{RC}}}+f_{D} e^{-\tilde{t} / \tilde{\tau}_{D}}\right)\right\}\right] d \tilde{x} .
\end{aligned}
$$

We integrate to obtain the long-time pressure. The integration constant is determined similar to the frequency dependent case:

$$
\tilde{p}-\tilde{p}_{0}=\frac{\cosh (2 \kappa L)}{2}\left(f_{x}-f_{x} e^{-\tilde{t} / \tilde{\tau}_{x}}\right)^{2}+\frac{1}{2}\left(f_{x}^{2} e^{-2 \tilde{t} / \tilde{\tau}_{x}}-2 f_{x}^{2} e^{-\tilde{t} / \tilde{\tau}_{x}}\right)+2\left[\cosh (\kappa L x)\left(f_{x}-f_{x} e^{-\tilde{t} / \tilde{\tau}_{x}}\right)\left(f_{\mathrm{RC}} e^{-\tilde{t} / \tilde{\tau}_{\mathrm{RC}}}+f_{D} e^{-\tilde{t} / \tilde{\tau}_{D}}\right)\right] .
$$

Thus we have the total force,

$$
\tilde{F}_{\text {tot }}=\tilde{p}-\tilde{p}_{0}-\frac{1}{2}\left(\frac{\partial \tilde{\phi}}{\partial \tilde{x}}\right)^{2}=-\frac{f_{x}^{2}}{4}-\frac{1}{2}\left(f_{\mathrm{RC}} e^{-\tilde{t} / \tilde{\tau}_{\mathrm{RC}}}+f_{D} e^{-\tilde{t} / \tilde{\tau}_{D}}\right)^{2} .
$$

Here the steady equilibrium value of the force is given by

and the dynamic part of the force is

$$
\tilde{F}_{\text {eq }}=-\frac{f_{x}^{2}}{4}=-\frac{\tilde{V}_{0}^{2}}{4} \kappa^{2} L^{2} \operatorname{cosech}^{2}(\kappa L),
$$

$$
\tilde{F}_{\text {dyn }} \equiv \tilde{F}_{\text {tot }}-\tilde{F}_{\text {eq }} \sim-\frac{1}{2}\left(f_{\mathrm{RC}} e^{-\tilde{t} / \tilde{\tau}_{\mathrm{RC}}}+f_{D} e^{-\tilde{t} / \tilde{\tau}_{D}}\right)^{2} .
$$

This corresponds to (13) in the main text.

[1] J. S. Newman and K. E. Thomas-Alyea, in Electrochemical Systems (John Wiley and Sons, Hoboken, NJ, 2012), p. 647.

[2] W. B. Russel, D. A. Saville, and W. R. Schowalter, Colloidal Dispersions (Cambridge University Press, Cambridge, UK, 1989).

[3] J. N. Israelachivili, Intermolecular and Surface Forces (Academic Press, New York, 2015).

[4] D. C. Prieve, Measurement of colloidal forces with TIRM, Adv. Colloid Interface Sci. 82, 93 (1999).
[5] W. A. Ducker, T. J. Senden, and R. M. Pashley, Direct measurement of colloidal forces using an atomic force microscope, Nature (London) 353, 239 (1991).

[6] M. Ayyildiz, M. Scaraggi, O. Sirin, C. Basdogan, and B. N. J. Persson, Contact mechanics between the human finger and a touchscreen under electroadhesion, Proc. Natl. Acad. Sci. USA 115, 12668 (2018).

[7] C. S. Perez-Martinez and S. Perkin, Surface forces generated by the action of electric fields across liquid films, Soft Matter 15, 4255 (2019). 
[8] M. A. Gebbie, M. Valtiner, X. Banquy, E. T. Fox, W. A. Henderson, and J. N. Israelachvili, Ionic liquids behave as dilute electrolyte solutions, Proc. Natl. Acad. Sci. USA 110, 9674 (2013).

[9] F. Li, D. P. Josephson, and A. Stein, Colloidal assembly: The road from particles to colloidal molecules and crystals, Angew. Chem. Int. Ed. 50, 360 (2011).

[10] A. Kumar, J.-S. Kwon, S. J. Williams, N. G. Green, N. K. Yip, and S. T. Wereley, Optically modulated electrokinetic manipulation and concentration of colloidal particles near an electrode surface, Langmuir 26, 5262 (2010).

[11] J. Israelachvili, Y. Min, M. Akbulut, A. Alig, G. Carver, W. Greene, K. Kristiansen, E. Meyer, N. Pesika, K. Rosenberg, and H. Zeng, Recent advances in the surface forces apparatus (SFA) technique, Rep. Prog. Phys. 73, 036601 (2010).

[12] P. M. Biesheuvel, Dynamic charge regulation model for the electrostatic forces between ionizable materials, Langmuir 18, 5566 (2002).

[13] S. Shulepov, S. Dukhin, and J. Lyklema, A dynamic approach to colloid particle interaction, J. Colloid Interface Sci. 171, 340 (1995).

[14] R. Podgornik, General theory of charge regulation and surface capacitance, Technical Report, 2018 (unpublished).

[15] J. W. Krozel, Electrokinetic interactions between two spheres: The role of surface charge transport in coagulation, J. Colloid Interface Sci. 163, 437 (1994).

[16] S. S. Dukhin and J. Lyklema, Dynamics of colloid particle interaction, Langmuir 3, 94 (1987).

[17] D. Weaver and D. Feke, Ageing in colloidal flocs: Effect of double-layer relaxation, J. Colloid Interface Sci. 103, 267 (1985).

[18] M. Z. Bazant, K. Thornton, and A. Ajdari, Diffuse-charge dynamics in electrochemical systems, Phys. Rev. E 70, 021506 (2004).

[19] J.-P. Hsu, Y.-C. Kuo, and S. Tseng, Dynamic interactions of two electrical double layers, J. Colloid Interface Sci. 195, 388 (1997).

[20] R. Tivony, S. Safran, P. Pincus, G. Silbert, and J. Klein, Charging dynamics of an individual nanopore, Nat. Commun. 9, 4203 (2018).

[21] M. Checa, R. Millan-Solsona, and G. Gomila, Frequencydependent force between ac-voltage-biased plates in electrolyte solutions, Phys. Rev. E 100, 022604 (2019).

[22] E. Spedden and C. Staii, Neuron biomechanics probed by atomic force microscopy, Int. J. Mol. Sci. 14, 16124 (2013).

[23] C. J. Bettinger, Recent advances in materials and flexible electronics for peripheral nerve interfaces, Bioelectron. Med. 4, 6 (2018).

[24] H.-C. Chang and G. Jaffé, Polarization in electrolytic solutions. Part I. Theory, J. Chem. Phys. 20, 1071 (1952).

[25] J. R. Macdonald, Theory of ac space-charge polarization effects in photoconductors, semiconductors, and electrolytes, Phys. Rev. 92, 4 (1953).
[26] B. Balu and A. Khair, Role of Stefan-Maxwell fluxes in the dynamics of concentrated electrolytes, Soft Matter 14, 8267 (2018).

[27] A. Sawada, Introduction of effective dielectric constant to the Poisson-Nernst-Planck model, Phys. Rev. E 93, 052608 (2016).

[28] G. Barbero and I. Lelidis, Effective dielectric constant of electrolytes, J. Appl. Phys. 115, 194101 (2014).

[29] M. M. Hatlo, R. Van Roij, and L. Lue, The electric double layer at high surface potentials: The influence of excess ion polarizability, Europhys. Lett. 97, 28010 (2012).

[30] G. Barbero and I. Lelidis, Evidence of the ambipolar diffusion in the impedance spectroscopy of an electrolytic cell, Phys. Rev. E 76, 051501 (2007).

[31] A. Poisson and A. Papaud, Diffusion coefficients of major ions in seawater, Mar. Chem. 13, 265 (1983).

[32] A. J. Bard and L. R. Faulkner, in Electrochemical Methods: Fundamentals and Applications (Wiley, New York, 2001), p. 833 .

[33] F. R. de Hoog, J. H. Knight, and A. N. Stokes, An improved method for numerical inversion of Laplace transforms, SIAM J. Sci. Stat. Comput. 3, 357 (1982).

[34] M. Mittal, P. P. Lele, E. W. Kaler, and E. M. Furst, Polarization and interactions of colloidal particles in ac electric fields, J. Chem. Phys. 129, 064513 (2008).

[35] J. P. Singh, P. P. Lele, F. Nettesheim, N. J. Wagner, and E. M. Furst, One- and two-dimensional assembly of colloidal ellipsoids in ac electric fields, Phys. Rev. E 79, 050401(R) (2009).

[36] J. Kim, J. L. Anderson, S. Garoff, and P. J. Sides, Effects of zeta potential and electrolyte on particle interactions on an electrode under ac polarization, Langmuir 18, 5387 (2002).

[37] T. J. Woehl, K. L. Heatley, C. S. Dutcher, N. H. Talken, and W. D. Ristenpart, Electrolyte-dependent aggregation of colloidal particles near electrodes in oscillatory electric fields, Langmuir 30, 4887 (2014).

[38] M. S. Kilic, M. Z. Bazant, and A. Ajdari, Steric effects in the dynamics of electrolytes at large applied voltages. II. Modified Poisson-Nernst-Planck equations, Phys. Rev. E 75, 021503 (2007).

[39] M. Z. Bazant, B. D. Storey, and A. A. Kornyshev, Double Layer in Ionic Liquids: Overscreening versus Crowding, Phys. Rev. Lett. 106, 046102 (2011).

[40] A. Levy, M. Bazant, and A. Kornyshev, Ionic activity in concentrated electrolytes: Solvent structure effect revisited, Chem. Phys. Lett. 738, 136915 (2020).

[41] M. Janssen, Curvature affects electrolyte relaxation: Studies of spherical and cylindrical electrodes, Phys. Rev. E 100, 042602 (2019).

[42] S. M. H. Hashemi Amrei, S. C. Bukosky, S. P. Rader, W. D. Ristenpart, and G. H. Miller, Oscillating Electric Fields in Liquids Create a Long-Range Steady Field, Phys. Rev. Lett. 121, 185504 (2018). 